\title{
Correction to: Reconstruction of AAOS type III and IV acetabular defects with the Ganz reinforcement ring: high failure in pelvic discontinuity
}

\author{
Cynthia Hourscht ${ }^{1} \cdot$ Mohammad K. Abdelnasser $^{1} \cdot$ Sufian S. Ahmad ${ }^{1}$ (1) $\cdot$ Lukas Kraler $^{1} \cdot$ Marius J. Keel $^{1}$. \\ Klaus A. Siebenrock ${ }^{1}$. Frank M. Klenke ${ }^{1}$
}

Published online: 19 December 2018

(c) Springer-Verlag GmbH Germany, part of Springer Nature 2018

\section{Correction to: \\ Arch Orthop Trauma Surg (2017) 137:1139-1148 \\ https://doi.org/10.1007/s00402-017-2731-x}

The author would like to correct the errors in the publication of the original article. The corrected details are given below for your reading.

The caption of Fig. 2 is not correct. The correct caption should be:

Radiographs of a female patient that underwent THA revision due to pelvic discontinuity at the age of 61 years. The AAOS IV defect was addressed with a Ganz reinforcement ring and posterior plating (a). Radiographs seven month after the reconstruction demonstrated failure of the reconstruction with dislocation of the Ganz reinforcement ring (b). The patient was revised again; reconstruction was performed with a Ganz reinforcement ring, structural and morselized bone grafts, and plating of the anterior and posterior column (c). After another 22 months, radiographs showed repeated failure as demonstrated by migration of the Ganz reinformcement ring, breakage of the anterior plate, and progressive loosening of the posterior plate (d). Due to critical illness of the patient, repeat revision surgery was not performed. The patient died four months after the diagnosis of the failure.

The correct ethical approval statement should be:

The study was approved by the ethical committee of the Canton on Bern, Switzerland (Ref.-Nr. KEK-BE: 265/2014).
The original article can be found online at https://doi.org/10.1007/ s00402-017-2731-x.

Sufian S. Ahmad

sufiansamy@gmail.com

1 Department of Orthopedic Surgery, Inselspital, Bern

University Hospital, Freiburgstrasse, 3010 Bern, Switzerland 